\title{
RESEARCH OF VISION-BASED NAVIGATION AND POSITIONING TECHNOLOGY FOR MOBILE CAMPUS INTELLIGENT ROBOT
}

\author{
Zhixian Yang ${ }^{1^{*}}$, Dong $\mathrm{Li}^{1}$ \\ ${ }^{1}$ Henan intelligent education and Intelligent Technology Application Engineering Technology Research Center, \\ Henan Province, China \\ *Corresponding author: Zhixian Yang, Email: yangzhixianbest1@163.com
}

\begin{abstract}
This paper mainly studies the autonomous positioning and navigation technology of intelligent robots. In the campus environment to perform security monitoring tasks, this article uses gps to achieve location tracking. Due to the loss of gps signal due to campus buildings and trees, the monocular vision is further selected as a robot-assisted positioning method, and two positioning methods are combined. Achieve effective location monitoring. On the other hand, the use of monocular visual perception of the road environment to achieve autonomous navigation, mainly to achieve the GPS positioning function, manual road sign positioning function, road area detection function. Therefore, the paper tests the artificial road sign location and road identification respectively. The test results show that the acquisition success rate is $96.5 \%$ and the average processing speed is 30.34 frames/s without occlusion interference.
\end{abstract}

Keywords: Monocular Vision; Positioning; Navigation; Road Recognition; Artificial Road Sign.

\section{Introduction}

The environment of mobile robots is both indoor and outdoor. The indoor environment is characterized by a short displacement of the motion and a weaker light. The outdoor environment has the characteristics of long motion displacement, large illumination variation, and complex environment [1]. In order to enable the mobile robot to achieve autonomous driving tasks in an indoor or outdoor environment, the mobile robot needs to have the ability to independently sense the external environment, and complete the self-positioning and navigation functions [2]. Among them, location estimation, road detection, map creation, and route planning are all key issues to be solved.

This paper will use mobile robot as the project platform, based on its autonomous driving demand, considering its particularity in the campus as a range of activities, select and implement a GPS-assisted visual positioning navigation scheme. In the project, the GPS positioning function, manual road sign positioning function, and road area detection function will be mainly implemented.

\section{Literature Review}

Foreign research on service robots began in the late 1960s. In 1972, Nils Nilssen and Charles Rosen of Stanford Research Institute developed a typical autonomous mobile robot, Shakey, which pioneered the research in the field of mobile robots. Research on real-time control of robot systems in complex environments. Yang et al. designed the robot ontology structure for campus inspection requirements, and adopted GPS navigation as the global path planning and fuzzy control algorithm as the robot combination control strategy for local path planning [3]. Hameed is a home robot developed by Probotics in 1999. It uses a two-wheel differential drive platform to perform feature matching by inputting a known environmental map to obtain environmental information [4]. Nong is also able to recognize sound signals in indoor environments and complete the transport of small objects [5]. Wei et al. designed the campus security robot car in combination with the campus security issues that are often concerned by the society. Su Yanbao and others designed a smart welcome robot for the campus, with a welcome etiquette and teaching practice function [6].

\section{Visual Positioning and Road Recognition Navigation Technology}

\subsection{Edge detection}

The Roberts operator, the sobel operator, and the prewitt operator in the classical edge detection operator are edge detection belonging to the first order differential [7]. The Canny operator and the Laplacian operator belong to the second-order differential edge detection method. They need to undergo filtering, enhancement, and edge detection 
multi-step processing. The processing is more complicated and slower [8].

Based on the consideration of the road detection requirements and implementation effects, this paper has been verified by experiments and uses the Canny edge detection method.

In this paper, the adaptive canny edge detection is performed after segmentation of the image based on the adaptive region growing method, and good results are obtained through comparative experiments. The steps of the adaptive Canny edge detection algorithm are as follows:

(1) Smoothing processing: smoothing processing is to eliminate noise processing on the input image $I(i, j)$ by a Gaussian filter, and output smooth and less noisy image $\mathrm{O}(\mathrm{i}, \mathrm{j})$, one of which is to perform an input image twice. The weighting of the Vigos kernel, and the other is the first-order directional derivative of the two-dimensional Gaussian function. When the noise filter is used, a two-dimensional Gaussian kernel convolution operation is performed. After the Gaussian kernel is solved, the normalization operation of the entire core is performed. The discrete one-dimensional Gaussian kernel function is as follows:

$$
K=e^{\left(-i^{2} / 2 \delta^{2}\right)} / 2 \pi \delta
$$

The discrete two-dimensional Gaussian function is as follows:

$$
K(i, j)=e^{\left(-\left(i^{2}+j^{2}\right) / 2 \delta^{2}\right)} / 2 \pi \delta^{2}
$$

The gradient vector is as follows:

$$
\nabla K(i, j)=\left[\begin{array}{ll}
\frac{\partial K(i, j)}{\partial i} & \frac{\partial K(i, j)}{\partial i}
\end{array}\right]
$$

The above equation is decomposed, and the obtained one-dimensional filter equation is as follows:

$$
\begin{aligned}
& \frac{\partial K}{\partial i}=i e^{\frac{-i}{2 \delta^{2}}} e^{\frac{-j}{2 \delta^{2}}} / 2 \pi \delta^{4} \\
& \frac{\partial K}{\partial j}=i e^{\frac{-j}{2 \delta^{2}}} e^{\frac{-i}{2 \delta^{2}}} / 2 \pi \delta^{4}
\end{aligned}
$$

Among them, the filter control smoothing parameters, the smoothed image $\mathrm{O}(\mathrm{i}, \mathrm{j})$ is as follows:

$$
o(i, j)=\nabla K(i, j) \bullet I(i, j)
$$

Gaussian filtering essentially uses a certain parameter criterion to combine the pixel values of the input image and the gray values of the surrounding points to perform weighted averaging to achieve high-frequency noise filtering.

(2) Calculation of amplitude and direction: edge detection is based on edge gradient detection, while gradient has two characteristics of amplitude and direction, which is the change of image chromaticity or brightness, and the gradient amplitude reflects the edge intensity of the image.
The gradient direction is the normal vector of the image. The gradient formula is as follows:

$$
F_{i}(i, j)=\partial F / \partial i \quad F_{j}(i, j)=\partial F / \partial j
$$

By simplifying the formula, the common formula for the gradient magnitude $M(\mathrm{i}, \mathrm{j})$ is as follows:

$$
M(i, j)=\left|F_{j}(i, j)\right|+\left|F_{i}(i, j)\right|
$$

The gradient direction of $M(\mathrm{i}, \mathrm{j})$ is obtained by first-order difference as follows:

$$
\theta(i, j)=\arctan \left(F_{i}(i, j) / F_{j}(i, j)\right)
$$

Among them, $\mathrm{Fi}(\mathrm{i}, \mathrm{j}), \mathrm{Fj}(\mathrm{i}, \mathrm{j})$ are gradients in the horizontal direction and the vertical direction. Gradient along the edge direction, the gradient amplitude does not change much, and is relatively flat. However, the gradient of the gradient in the direction of the vertical edge becomes larger and stronger [9].

\subsection{Road segmentation}

Nowadays, there are region segmentation, threshold segmentation, hybrid algorithm segmentation, etc., where the region growing method (srg) is a hybrid segmentation method. In general, the area segmentation method is better than other segmentation methods in terms of noise immunity. The region growth method relies on the definition of the initialization seed and the growth pixel region growth criterion. When the target region and the background are clearly distinguished, the growth is performed. The criterion is better defined. In this case, the method will get better segmentation effect. In this paper, the distinction between the color and the background of the road region is more obvious. The segmentation method based on the region growth method will be used to segment the road image.

There are two main parameters of traditional regional growth: the global region average gray value and the global region average gradient. Then this parameter selection is not well suited for good environmental changes. Based on the environmental variability of the acquired road image, this paper uses the adaptive region growing method for road segmentation. The two key steps are as follows:

(1) Selection of seed points: These two parameters are changed from the global region to the local region, and the local region average gray level and average gradient are calculated. Then these two parameters are determined by the pixel points, the number of iterations, and the local area. Since the selection of seed points is crucial, people often propose different optimizations. In the traditional area growing method, a single pixel is generally selected as the initial seed, but since the picture has various types of noise, the selected seed point may be a miss-selection of the noise point, so this paper 
selects the $x$ pixel point $3^{*} 3$ neighbour. The seed region of the domain is used as the initial seed, and this type of situation can be appropriately avoided [10].

Define the grown region as $\mathrm{R}$, define the local average gray value $H(x)$ of the point $x$, the $3 * 3$ neighbourhood of the pixel point $x$ is defined as $O(x)$, and the pixel point $\mathrm{Z}$ is any point in $\mathrm{O}(\mathrm{x}), \mathrm{Oin}(\mathrm{x})$ is a coincident region of the $3^{*} 3$ neighbourhood of the grown region and the pixel point $\mathrm{x}$, the point $\mathrm{y}$ is an arbitrary pixel point of the coincident region class, and $F(y)$ is the gray value of the pixel point $y, S O$ in $(\mathrm{x})$ is the sum of the pixels of the coincident region, and $\operatorname{So}(z)$ is the sum of all the pixels of the neighbourhood of the pixel point $Z$. Then the local region average gray level $\mathrm{H}(\mathrm{x})$ and the local average gradient are calculated as follows:

$$
\begin{aligned}
& H(X)=\sum_{Y \in O_{i n}(X)} F(Y) / S_{O_{i n}(X)} \\
& \|\nabla\|_{X}=\sum_{Z \in O(X)} F(Z) / S_{O(X)}
\end{aligned}
$$

(2) Similarity criterion: In the regional growth method, the growth rule is another key factor. The selected growth rule is related to the result of subsequent growth. According to the local parameters, we adopt the adaptive similarity criterion as follows:

$$
H(X)-m \bullet\|\nabla\|_{X}<F(Y)<H(X)+n \bullet\|\nabla\|_{X}
$$

It can be seen from the above equation that the gray value of the pixel point $y$ can vary within a range, but when its pixel gray value exceeds this range, the region that does not belong to the similarity is to be rejected. Pixels within this range will be summarized as similar regions that satisfy the condition until the last condition is not met.

It can be seen from the road segmentation realization map analysis based on the growth region method in the following figure that the method can clearly distinguish the road region from the surrounding irrelevant region, and can eliminate the interference of the false edge, and achieve the demand of the road segmentation part of the project.

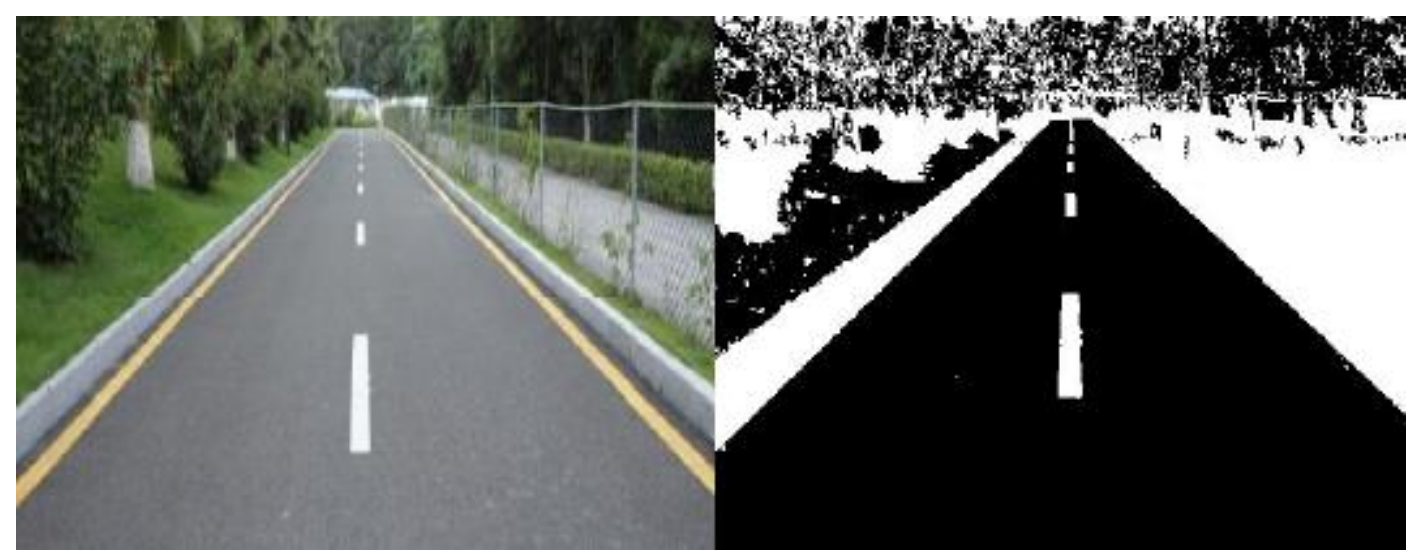

Figure 1: Processing effect of road segmentation method based on adaptive growth region method

\subsection{Line detection}

Since the detected road edge line is not smooth, there is a noise point. We first use the mathematical morphology method to solve this problem, and the road edge is compensated by a small number of breakpoints or unsmooth areas after multiple expansion and corrosion operations. Perform line detection.

Based on the road special case, and the continuous straight line portion in the road image after the road segmentation edge extraction is extracted, much interference information is reduced relative to the unprocessed. The road line is obvious, but the robot's motion environment has more interference information, and the hough transform has stronger anti-interference ability. This paper uses the hough transform as a line detection method.

Since the campus road is a structured road, the road line is generally white or yellow, and the road surface is mainly gray. The color contrast of the lane line and the road area is obvious, because the lane line of the road generally has a certain width, and both There are roadside edges. In real life, there are external factors such as road line wear and human vehicle occlusion, and Hough change only determines the straight line according to the number of points on the straight line, which may result in a false lane line. Therefore, this paper proposes a Hough edge line detection method combined with conditional constraints in combination with the actual situation of the security robot's working environment.

(1) Constraint based on road line direction

After analyzing and observing the results of road segmentation and edge detection, it can be seen that some of the extracted edge information are not road edges, so some constraints can be added. In this paper, the Hough transform based on the directionoptimized constraint is used after the region is grown. The edge information has been saved in the edge map. The general road is divided into two parts of the road line. The left and right sides are symmetrically related, and the boundary of the left 
and right road areas is constrained based on the facts on the left and right boundary directions. It is assumed that the road line is clockwise along the $\mathrm{x}$ axis. In the negative direction and the counterclockwise direction, the angle of the left road edge line is in $[10,80]$, and the angle of the right lane edge line is $[100,170]$.

(2) Based on image region of interest constraints

Observing the video image information collected by the security robot, it can be seen that nearly $1 / 3$ of the image information in the image is information related to the road, the sky and the like. Not only does it have no auxiliary effect on road edge detection, but it also increases the computational burden in image processing of image edge detection. So, we don't need to calculate the entire image, just the real road area, which is the lower part of the image (2/3 image). With such conditional constraints, the road detection algorithm is greatly optimized.
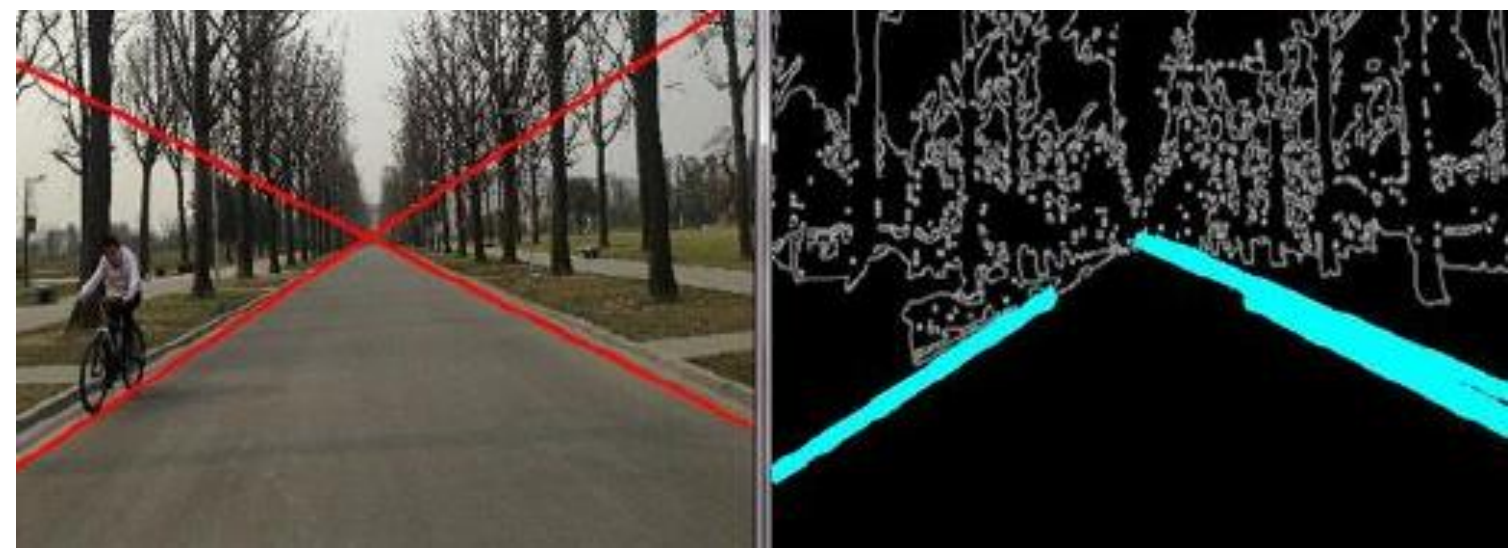

Figure 2: Hough transform line detection

\section{Actual System Construction}

\subsection{Project platform and hardware facilities}

This paper is based on the research and application of positioning and navigation technology on the security robot platform.

The traditional monitoring system generally fixes the camera to a certain corner to monitor the surrounding environment.

This type of monitoring range has limitations and cannot monitor the range of mobility.

And the installation is troublesome and difficult to transfer, and the project uses the camera device carried by the mobile robot to carry out real-time monitoring of the flow, and strives to achieve global monitoring of the community or the campus; the traditional community monitoring system requires the staff to observe in real time, and this project uses road identification to allow the security robot to autonomously monitor the community or campus under non-human intervention conditions, and send out alarm signals to the background in real time when dangerous situations (fire, smoke, suspicious people) are detected.

The entire security robot includes a mechanical body, an Android development board, an infrared camera, and a gps signal receiver.

The Android development board is an intermediate medium for positioning navigation.
The road camera and manual road sign information are obtained through the infrared camera to realize road area judgment and manual road sign positioning, and the Android development board sends commands to control the robot to perform walking, deflection stop and the like.

\subsection{Positioning navigation system}

The software includes five modules for offline map download, map creation, manual driving, automatic driving, and historical trajectory. The offline map download module in the software is the premise of other functional modules.

Before using other functional modules, you need to download the map of the city area. Offline map download provides the basic map for the other three modules; creating the map module is the key module of the first phase of the project.

In a new area, the map of the place must be created before the subsequent functions can be realized. And also need to send the track to the background server; the historical track records the various tracks in the process of creating the map for easy viewing; automatic driving is the key module of the second phase of the project, the module needs to interface with the robot terminal, plan the action path for the robot and pre. The path is commanded to control the robot; the manual driving module mainly plays an auxiliary role, and the main function is to plan the path and navigate. 


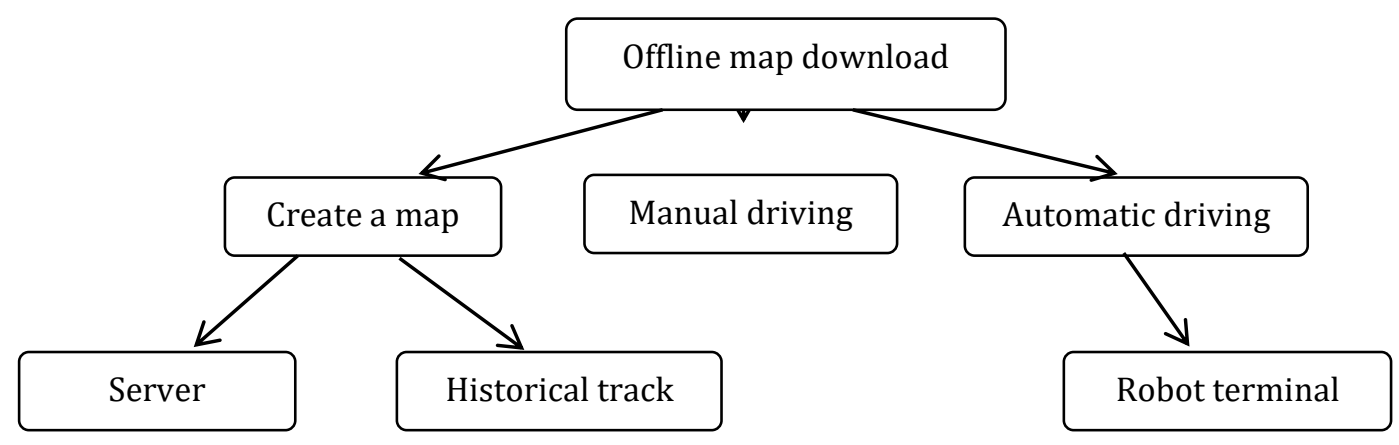

Figure 3: Overall structure

\section{Independent Driving Test}

\subsection{Artificial road sign positioning experiment test}

The experiment of manual road sign positioning is carried out indoors. Due to the wide outdoor view, the artificial road sign we need is a square outline mark with a certain size. Since there is no large artificial road sign for the outdoor product, we print it with a4 paper. Artificial road signs are taken and tested indoors.

The experiment contains the images of the two schemes designed in the paper.

This experiment mainly realizes the positioning of the artificial landmarks, which provides the premise for the subsequent image matching or decoding steps. We collect multi-directional and multi-angle images of simple QR codes and character road signs, including positions in the upper left corner, upper right corner, middle, lower right corner, long distance, close distance, offset, rotation, backlight, glare, etc.

The omni-directional test mainly includes the following test contents: 1) long-distance functional test; 2) offset functional test; 3) backlight functional test; 4) glare functional test; 5) vertical distortion Functional testing; 6) performance testing.

By testing the character road signs from the vertical angle, long distance, and illumination, the test is carried out. It is found that the double contour edges can be positioned in three aspects: backlight, redundant interference, and vertical angle. When the distance is far, the first position can only be located. The outer contour can be detected as the distance is asymptotic.

The two aspects of the front and the tilt of the coded road sign were tested, and it was found that both the tilt and the positive orientation can obtain a good positioning effect.

Table 1. Artificial road marking area detection

\begin{tabular}{|c|c|c|c|c|c|c|}
\hline $\begin{array}{c}\text { Testing } \\
\text { environment }\end{array}$ & $\begin{array}{c}\text { Number of } \\
\text { acquisitions } \\
\text { (frame) }\end{array}$ & $\begin{array}{c}\text { External } \\
\text { leak } \\
\text { detection } \\
\text { (frame) }\end{array}$ & $\begin{array}{c}\text { Internal } \\
\text { leak } \\
\text { detection } \\
\text { (frame) }\end{array}$ & $\begin{array}{c}\text { Missed } \\
\text { inspection } \\
\text { (frame) }\end{array}$ & $\begin{array}{c}\text { Misdetecti } \\
\text { on } \\
\text { (frame) }\end{array}$ & $\begin{array}{c}\text { Processing time } \\
\text { (millisecond) }\end{array}$ \\
\hline $\begin{array}{c}\text { Environment 1 } \\
\text { (backlight) }\end{array}$ & 180 & 7 & 10 & 2 & 0 & 5667 \\
\hline $\begin{array}{c}\text { Environment 2 } \\
\text { (sunshine) }\end{array}$ & 180 & 1 & 3 & 0 & 0 & 5682 \\
\hline
\end{tabular}

In order to test the positioning function of the artificial road sign area, the video images from far to near are collected in two indoor environments, and the first 180 frames of the two environments are analyzed respectively. The area starts counting, and the end number is counted as 180 frames. If the frame area in this period is not detected, the landmark area can be counted as a missed detection frame. When the proximity is entered, the determination is started. If the outer contour is detected, the external detection is performed. If the inner contour is detected, it is an internal leak detection, and all of them are detected as missing detection, and the landmark area positioning error is an error detection frame. The processing time is started from the first frame, and the timing is ended after 180 frames. In the environment 1, the near range is entered from the 84th frame, and the inner and outer double contours are detected. In environment 2, the short-range range is entered from 75 frames, and the inner and outer double contours are detected and positioned in the road marking area. The detection failure frame for the 180 -frame image in the backlight environment is 21 frames, and the detection failure frame for the 180frame image in the backlight environment is 4 frames, and the total failure rate is 25 frames in 300 frames, the failure rate is $6.94 \%$. The processing time of the environment, the average processing speed is 31.8 frames / $\mathrm{s}$. 


\subsection{Road identification test}

In this paper, the road area is mainly used for video sampling in the middle of the road and the right side of the road. 150 frames are collected in the middle of the road and the right side, and the captured video is compressed. The video image size is $320 * 240$, video 30 frames/s. In the road area detection experiment, in the image of one frame, the left edge is not displayed linearly to the left side, the right edge is not displayed as the right side, and the left and right lines are displayed. If there is a straight line that detects a straight line that is not at the edge of the road, it is a wrong check.

Table 2. Road area detection on the right side of the road

\begin{tabular}{|c|c|c|c|c|c|c|c|}
\hline \multicolumn{2}{|c|}{ Testing environment } & $\begin{array}{c}\text { Capture } \\
\text { picture } \\
\text { (frame) }\end{array}$ & $\begin{array}{c}\text { Missing } \\
\text { left side } \\
\text { (frame) }\end{array}$ & $\begin{array}{c}\text { Missing } \\
\text { right side } \\
\text { (frame) }\end{array}$ & $\begin{array}{c}\text { Left and } \\
\text { right } \\
\text { missed } \\
\text { detection } \\
\text { frame) }\end{array}$ & $\begin{array}{c}\text { Misdetection } \\
\text { (frame) }\end{array}$ & $\begin{array}{c}\text { Processing } \\
\text { time } \\
\text { (milliseconds) }\end{array}$ \\
\hline \multirow{2}{*}{$\begin{array}{c}\text { interme } \\
\text { diate }\end{array}$} & $\begin{array}{c}\text { cloudy } \\
\text { day }\end{array}$ & 150 & 1 & 1 & 0 & 0 & 4676 \\
\cline { 2 - 8 } sunlight & 150 & 0 & 0 & 0 & 0 & 5139 \\
\hline \multirow{2}{*}{ Right } & sunlight & 150 & 12 & 1 & 0 & 0 & 4690 \\
\cline { 2 - 9 } & $\begin{array}{c}\text { cloudy } \\
\text { day }\end{array}$ & 150 & 0 & 0 & 0 & 0 & 4930 \\
\hline \multirow{2}{*}{ Right } & $\begin{array}{c}\text { Vehicle } \\
\text { occlusion }\end{array}$ & 150 & 31 & 0 & 0 & 0 & 5279 \\
\hline \multicolumn{2}{|c|}{ Comprehensive } & 750 & 51 & 2 & 0 & 0 & 24714 \\
\hline
\end{tabular}

The right side of the road is close to the quarter of the road for data collection. The selected road width is narrower than the above-mentioned road width in the middle of the road. A total of 750 frames are collected, and 300 frames of data are used for the middle road and the right road. The experimental results show that 21 of the 600 frames acquired without failure of occlusion are the failure frames, the success rate is $96.5 \%$, and 32 frames are lost in 150 frames under the occlusion condition of the car. Most of them are frames with the car body close to the camera. The successful detection rate under this condition is $78.6 \%$.

The total processing time and the total number of frames under the five conditions in Table 2 can be obtained. The average processing speed is 30.34 frames/s, and the average time for processing one frame is about 32.95 milliseconds. Since the pal TV standard is 25 frames/s, that is, the processing time per frame should not exceed 40 milliseconds, the comprehensive test results basically meet the realtime requirements.
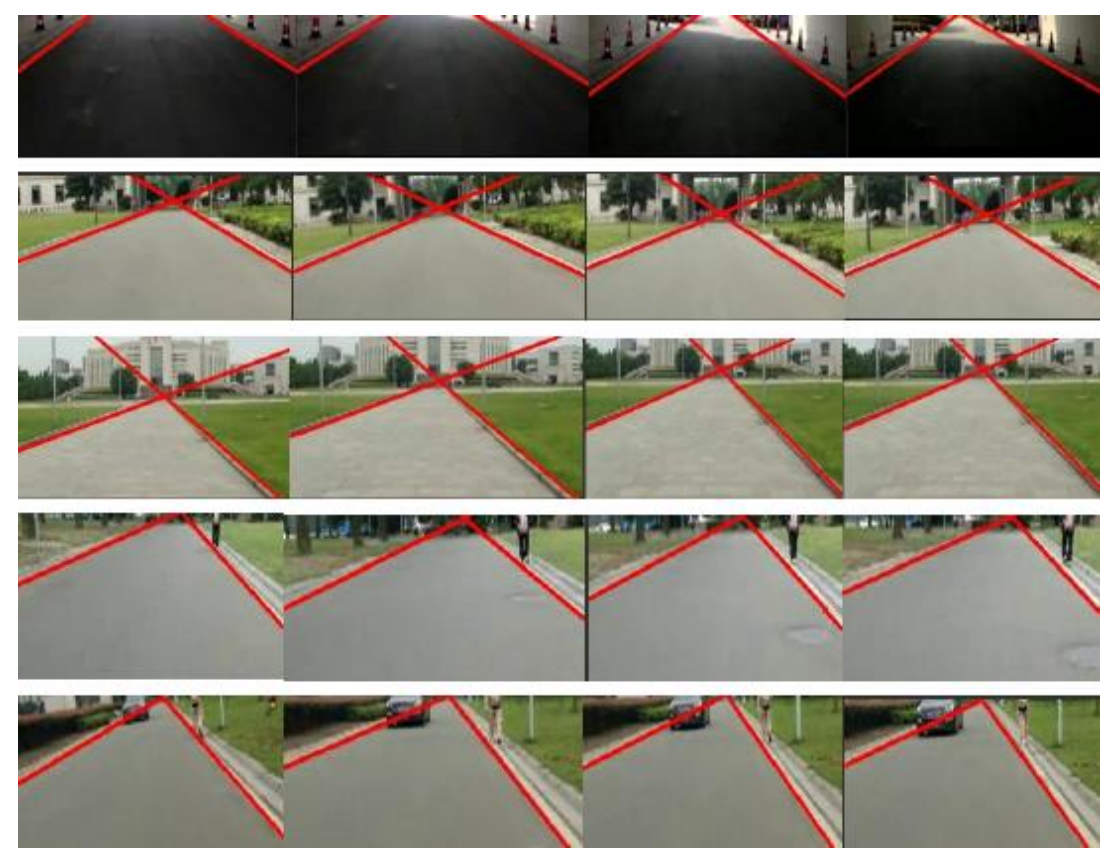

Figure 4: Road area detection performance test 
Based on the analysis and observation of the road recognition experiment results under various conditions, this paper is feasible for the road identification scheme of the structured road of the campus. In general, the road detection system implemented in this paper has good stability and real-time performance.

\section{Conclusions}

This paper takes mobile robot as the project platform, and based on its autonomous driving demand, considering its particularity in the campus as a range of activities, it selects and implements a GPS-assisted visual positioning navigation scheme. In the project, the gps positioning function, the manual road sign positioning function, and the road area detection function are mainly realized. The manual road sign location and road recognition were tested respectively. The test results show that the acquisition success rate is $96.5 \%$ and the average processing speed is 30.34 frames/s without occlusion interference. The overall satisfaction of real-time needs, the program is feasible.

\section{References}

[1] Tian, R., \& Li, Q. (2016). Research on the application of rectangle object constraint in active vision of mobile robot. International Conference on Robotics \& Automation Engineering.

[2] Hua, B., Rama, E., Capi, G., Jindai, M., \& Tsuri, Y. (2017). Intelligent navigation and accurate positioning of an assist robot in indoor environments. International Conference on Robotics \& Machine Vision.

[3] Yang, G., Zheng, X., Hui, C., \& Ling, H. (2017). Planar-based visual positioning for a mobile robot with monocular vision.

[4] Hameed, I. A. (2017). Using natural language processing (NLP) for designing socially intelligent robots. Joint IEEE International Conference on Development \& Learning \& Epigenetic Robotics.

[5] Nong, Q. (2017). Practical Secure Certificateless Cryptographic Protocol with Batch Verification for Intelligent Robot Authentication. International Conference on Mechatronics \& Intelligent Robotics.

[6] Wei, H., Li, Z., \& Chen, C. L. P. (2017). A survey of human-centered intelligent robots: issues and challenges. IEEE/CAA Journal of Automatica Sinica, 4(4), 602-609.

[7] Li, Z., Chen, C. L. P., \& Wei, H. (2017). Call for papers - special issue on human-centered intelligent robots: issues and challenges. IEEE/CAA Journal of Automatica Sinica, 3(4), 1-1.

[8] Maia Alexandre, F. (2017). The legal status of artificially intelligent robots: personhood, taxation and control. Social Science Electronic Publishing.

[9] Kefer, M., \& Tian, J. (2017). An intelligent robot for flexible quality inspection. IEEE International Conference on Information \& Automation.

[10] Zuhrie, M. S., Munoto, Hariadi, E., \& Muslim, S. (2018). The design of artificial intelligence robot based on fuzzy logic controller algorithm. IOP Conference Series: Materials Science and Engineering, 336, 012020. 